\title{
A Model of Measuring the Evolution and Influence in Society
}

\author{
Jixing Liu \\ School of North China Electric Power University, Baoding 071000, China; \\ 913374693@qq.com
}

Keywords: Society's information network, SIR epidemic model, MATLAB simulation

\begin{abstract}
With the rapid development of communication technology, flow of information has been accelerated time after time. Accompanied by the change, more attention is also paid to evolution and influence in society's information networks. Aiming to evaluate the speed and direction of information flow, the thesis utilizes differential equations to formulate the model. In the model, members of society are seen as nodes in the network. Motivated by SIR epidemic model, the thesis analyzes different nodes' features in the process of information flow and numerical development trends. On the basis of the above analysis, MATLAB is utilized to simulate the extent of information sharing when information flow reaches the steady state. The thesis further discusses its potential influence on public interest and opinion combined with data. Several suggestions are put forward on optimizing the social's information network.
\end{abstract}

\section{Introduction}

In the history of humanity development, the method of information communication has been changed over and over again. Humanity has gone through the eras whose most advanced technology was newspaper, telegraph, radio, television and the Internet respectively.

Nowadays, the public are able to attain a large amount of information, but the value of the information itself is well worth considered. At the same time, the speed and direction of information flow are complex. In some cases, urgent information may spread on a large scale rapidly; however, useful information is likely to be blocked in the process of flow because it cannot find appropriate media.

Therefore, how to make the flow of information identical to our expectations is undoubtedly important. Cognizing the transformation law of information flow scientifically becomes an essential type of research.

\section{Model}

Terminology and Definition. Table 1. Terminology and definitions Variable Description

\begin{tabular}{|c|c|}
\hline$v_{i}$ & node $i$ \\
\hline$k_{i}$ & the degree of node $i$ is $k$ \\
\hline$O_{i j}$ & the number of common neighbors \\
\hline
\end{tabular}

observation variable coefficient 


$\begin{aligned} & b_{i j} \\ & r(t) \\ & m\end{aligned}$
$\begin{aligned} & S_{k}(t) \\ & I_{k}(t) \\ & R_{k}(t) \\ & \text { the proportion of spreaders in the network } \\ & \lambda\end{aligned} \quad$ the proportion of ignorants in the network
$\lambda$

The foundation of model. In the model, nodes stand for people in the social network and whether the edge exists or not depicts the situation of information flow.

It is obvious that the stronger a kind of media's influence is, the more audience it will have. Thus the number of common neighbors for node $i$ and node $j\left(O_{i j}\right)$ is related to the media's influence power $(\zeta)$, as well as the used amount of the media( $X)$. After reviewing related literature, we can attain the equation as follows[1]:

$$
O_{i j}=\delta X
$$

In order to estimate $\zeta$, four factors are introduced to describe: the duration of information flow $\left(X_{1}\right)$,the extent of media interaction $\left(X_{2}\right)$, the number of the audience $\left(X_{3}\right)$ and the location of information source $\left(X_{4}\right)$.Delphi method is used to evaluate the weight and proceed the normalization processing. Delphi method is a way of social investigation to deal with data, making the range of $X_{1} \sim X_{4}$ is $0 \sim 1$. The observation variable coefficient $(\eta)$ and observation $\operatorname{error}(\delta)$ are utilized to reduce error[1]. The equation can be represented as

$$
\zeta_{i}=\frac{X_{i}}{\eta_{i}}+\delta
$$

When the sample number reaches a high level, the value of $\delta$ is so tiny that it can ignored. Table 2 shows the values of $\eta_{i}$ when $X_{i}$ are different. To attain more accurate results, the value of $\zeta_{i}$ in calculation has been averaged.

Table 2. $\eta_{i}$ for different $X_{i}$

\begin{tabular}{lcccc}
\hline$X_{i}$ & $X_{1}$ & $X_{2}$ & $X_{3}$ & $X_{4}$ \\
$\eta_{i}$ & 0.94 & 1.00 & 1.03 & 1.02 \\
\hline
\end{tabular}

Through the above analysis, the value of $O_{i j}$ by calculation can be worked out. It is apparent that if the larger the number of our common neighbors is, the proportion of common friends $\left(w_{i j}\right)$ is higher. Thus, $w_{i j}$ is closely related to $O_{i j}$ and we have the below formula[2]: 


$$
w_{i j}=\frac{O_{i j}}{k_{i}-1+k_{j}-1-O_{i j}}
$$

(2) It can be

proposed that the concept of interpersonal reinforcement strength $\left(b_{i j}\right)$ can indicate the intimating degree of nodes from the perspective of sociology. Linear threshold uses $\frac{1}{k_{j}}$ to represent $b_{i j}$, which means the effects of each node connected to node $i$ on $j$ is all the same. Obviously, this assumption neglects the intimating degree among members in the social network, which isn't identical to the reality. Therefore, we introduce the proportion of common friends into the formula. Its form is showed in the below way[3]:

$$
b_{i j}=\frac{w_{i j}}{\operatorname{Max}_{k} \in \Gamma\left(W_{i k}\right)}
$$

In the formula, $\Gamma_{j}$ represents the set of all nodes connected to node $i$. When an ignorant node $j$ receives the information from node $i$ at the time of $t$, frequency of node $j$ 's receiving information is $r(t)$.The formula for the probability that node $i$ accepts the information $(\lambda)$ is as follows[3]:

$$
\lambda=1-e^{-\left(m(r(t)-1)+b_{i j}\right)}
$$

The formula shows that whether node $j$ accepts the information and becomes a new spreader or not has no relationship with the first $r$-1 frequency of flow. Instead, it is only related to the edge and direction of information flow at the frequency of $r$. Besides, the appeal of information $(m)$ also makes effects on $\lambda$.

According to the common sense, when new information appears, few people knows it. Reflecting it in the model, we think that the proportion of spreaders $\left(S_{k}(0)\right)$ is little at the initial time and remaining people are all ignorants. The proportion of removals is zero. The numbers of nodes in the society's information network is $N$, and equations of initial conditions are expressed as:

$$
\left\{\begin{array}{l}
S_{k}(0)=\frac{1}{N} \\
I_{k}(0)=\frac{N-1}{N} \\
R_{k}(0)=0
\end{array}\right.
$$

When information starts to flow in the network, the development trend of nodes' proportion has two types of possibilities:

- When a spreader deliver information to an ignorant, the ignorant may accept the information and become a new spreader if he is interested in the information and believes in the information's authenticity. The occurrence probability of this circumstance is $\lambda$. Otherwise, he will refuse to accept the information and become a removal.

- When a spreader deliver information to a removal or spreader, he may refuse to re-accept the information. Under this circumstance, he will not spread the information either. The occurrence probability of this circumstance is $\alpha$. We set $\alpha$ as a constant. 
In view of the above discussion, we can propose the following set of coupled differential equations about information flow on the basis of inhomogeneous networks[4]:

$$
\left\{\begin{array}{l}
\frac{d I_{k}(t)}{d t}=-\lambda\left(w_{i j}\right) k I_{k}(t) \sum_{k^{\prime}} \frac{k^{\prime} p\left(k^{\prime}\right) S_{k^{\prime}}(t)}{\langle k\rangle} \\
\frac{d S_{k}(t)}{d t}=\lambda\left(w_{i j}\right) k I_{k}(t) \sum_{k} \frac{k^{\prime} p\left(k^{\prime}\right) S_{k^{\prime}}(t)}{\langle k\rangle}-\alpha k S_{k} \sum_{k} \frac{k^{\prime} p\left(k^{\prime}\right)\left[S_{k^{\prime}}(t)+R_{k^{\prime}}(t)\right]}{\langle k\rangle} \\
\frac{d R_{k}(t)}{d t}=\alpha k S_{k}(t) \sum_{k^{\prime}} \frac{k p\left(k^{\prime}\right)\left[S_{k^{\prime}}(t)+R_{k^{\prime}}(t)\right]}{\langle k\rangle}
\end{array}\right.
$$

In the above equations, $p\left(k^{\prime}\right)$ is actually conditional probability indicating the connection intensity between node $i$ and node $j$, whose degree is $k$ and $k^{\prime}$ respectively. The formula for $p\left(k^{\prime}\right)$ is as follows[5]:

$$
p\left(k^{\prime}\right)=\frac{w_{i j}}{\sum_{j \in \Gamma_{j}} w_{i j}}
$$

Then we add the supplementary equation. The supplementary equation is constantly valid during the process of information flow. We can get

$$
S_{k}(t)+I_{k}(t)+R_{k}(t)=1
$$

\section{Discussion}

Information sharing. The above simulation has demonstrated that the majority of social members can share the information. Aiming to evaluate information sharing intuitively, we utilize MATLAB to simulate the connected situation between nodes. We assume that the numbers of nodes in the society's information network $(N)$ is 10 , and the time of information flow is 100 , and the appeal of information $(m)$ is 0.5 .

The simulation results are exhibited in the following Figure 1.

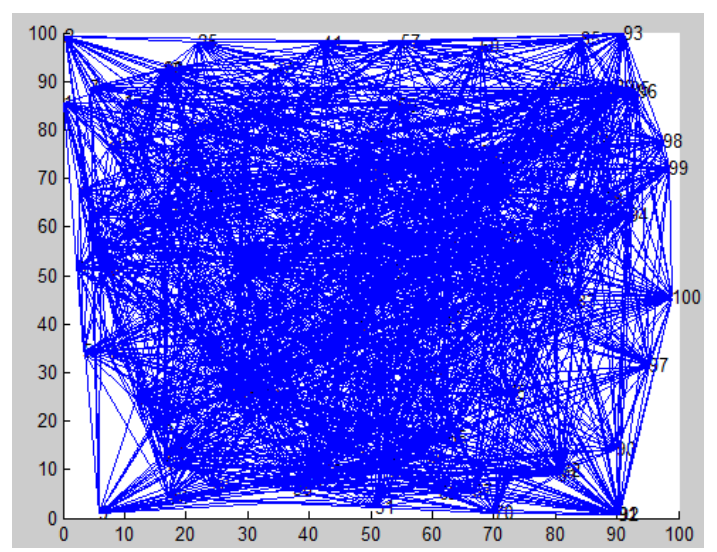

Figure 1. The connected situation between nodes in the network

Figure 1 demonstrates that nearly every nodes in the social network has the chance to accept the information. During the flow of information, the extent of information sharing is always rising. That means useful information is open to the public. Everyone has access to know about and take 
advantage of the information. If so, the gap of knowledge level can be eliminated one day. Society will also be more fair, honest and harmonious.

\section{References}

[1] Shen Yang and Wu Jingji: Public opinion deduction based on complexity factors. Journal of the China Society For Scientific And Technical Information.2013.p.1315.

[2] J.P.Onnela,Ki.J.Saram: Structure and tie strengths in mobile communication networks. vo.104, no.18,pp.7332.2007.

[3] Cheng Junjun: Research on information dissemination and topics growth trends prediction in social networks.2013.

[4] Y.Moreno, M.Nekovee, A.F.Pacheco: Dynamics of rumor spreading in complex networks.Phys.Rev.E,2004(69): 066130. 